\title{
THE RELATIVE FORCE IN SQUAT JUMP IS THE BEST LABORATORY PREDICTOR OF SPRINT PERFORMANCE IN ADOLESCENT SOCCER PLAYERS
}

original paper

doi: https://doi.org/10.5114/hm.2017.73622

\author{
LADISLAV DRAGULA ${ }^{1}$, MICHAL LEHNERT ${ }^{1}$, RUDOLF PSOTTA ${ }^{1}$, ZUZANA GONOSOVÁ ${ }^{1}$, \\ SVATOSLAV VALENTA ${ }^{1}$, PETR ŠTASTNÝ ${ }^{2}$ \\ ${ }^{1}$ Faculty of Physical Culture, Palacký University, Olomouc, Czech Republic \\ ${ }^{2}$ Faculty of Physical Education and Sport, Charles University, Prague, Czech Republic
}

\begin{abstract}
Purpose. The aim was to determine the relationships between linear sprint performance and different outcomes relating to isokinetic strength, jump performance, and intermittent Wingate test performance in well-trained young (U19) soccer players.

Methods. The total of 18 elite male soccer players (age: $17.6 \pm 0.8$ years; body mass: $73.3 \pm 5.9 \mathrm{~kg}$, height: $180.8 \pm 4.8 \mathrm{~cm}$ ) performed a 20-m sprint test, countermovement jump, squat jump (SqJ), isokinetic measurement of knee flexors and extensors strength, and intermittent anaerobic test on a bicycle ergometer (IAnTBE10 $\times 6$ s with a 30-s rest interval). The strength and power outcomes of laboratory tests served as possible predictors of sprint performance in multiple regression analyses. Results. The correlation analyses showed a significant relationship between all sprint lengths and relative maximum force measured by SqJ ( $r$ value: from -0.48 to -0.67$)$ and between all sprint lengths and peak power (PP) measured by IAnTBE10 $\times 6 \mathrm{~s}$ ( $r$ value: from -0.47 to -0.55$)$. The average power measured by IAnTBE10 $\times 6 \mathrm{~s}$ was associated with sprint lengths of 10,15 , and $20 \mathrm{~m}$ ( $r$ value: from -0.45 to -0.49$)$. The SqJ predicted the $5-\mathrm{m}$ sprint $\left(R=0.48 ; R^{2}=0.18\right)$ and $10-\mathrm{m} \operatorname{sprint}\left(R=0.55 ; R^{2}=0.26\right)$. The PP together with the relative force (RF) predicted the $15-\mathrm{m}\left(R=0.65 ; R^{2}=0.51\right)$ and $20-\mathrm{m}\left(R=0.77 ; R^{2}=0.64\right) \mathrm{sprint}$ performance.
\end{abstract}

Conclusions. The RF in SqJ can be used as a parameter relevant to linear 5-m, 10-m, 15-m, and 20-m sprint. Together with PP, it can be applied as a parameter relevant to linear 15-m and 20-m sprint.

Key words: acceleration, regression analyses, muscle strength, football

\section{INTRODUCTION}

Generally, laboratory physical performance tests designed for athletes are useful for planning or modification of a training programme only when they are of sufficient validity related to the specificity of a particular sport [1]. Elite European soccer players cover $1.8-2.6 \%$ of the total match distance by sprinting [2]. During a soccer game, sprinting occurs every $90 \mathrm{~s}$, lasting on average $2-4 \mathrm{~s}$. Therefore, the ability to accelerate during very short sprints is important to succeed in soccer [3]. Moreover, sprinting abilities discriminate between soccer player performance levels $[4,5]$. Thus, in sports practice, when designing a training programme focused on the sprinting ability or assessing its effect, the results of not only field tests but also laboratory tests could be useful. The main advantage of laboratory tests is that they provide profound variable-related information at a mechanical and physiological level [1]. Consequently, it is important for coaches and other specialists in soccer teams to know which laboratory variables may predict sprint abilities in soccer players.

Therefore, there is a question which neuromuscular functions or abilities relate to sprint performance in the first acceleration phase of sprinting, and which laboratory tests might capture those functions with efficient validity. It has been documented that sprint

Correspondence address: Petr Stastny, Department of Sport Games, Charles University, Jose Martiho 31,16252 Prague,

Czech Republic, e-mail: stastny@ftvs.cuni.cz

Received: December 15, 2017

Accepted for publication: January 24, 2018

Citation: Dragula L, Lehnert M, Psotta R, Gonosová Z, Valenta S, Štastný P. The relative force in squat jump is the best laboratory predictor of sprint performance in adolescent soccer players. Hum Mov. 2017;18(5)special/issue:83-90; doi: https:// doi.org/10.5114/hm.2017.73622. 
L. Dragula et al., Squat jump as a predictor of sprint in soccer

performance is determined by a number of constraints, such as thigh muscle strength [6-8] and lower limb explosive abilities [9-11]. Previous studies reported an association between the countermovement jump (CMJ), squat jump (SqJ) or full squat and 5-m [12], 10-m [12, 13], 20-m [12, 13], and 30-m [13] sprint. Furthermore, sprint performance over $5,10,20$, and $30 \mathrm{~m}$ showed a relationship with isokinetic knee flexion and isokinetic knee extension [14-16]. The main reason why the isokinetic hamstring strength has a potential to be a sprint performance predictor is that during sprinting the hamstring plays an important propulsive role and assists the quadriceps in hip extension in the push-off phase [17-19]. Therefore, the neuromuscular functions such as isokinetic strength or net force production during jumping might be good predictors of short sprint performance.

Regarding a large number of available laboratory tests, test devices, and protocols that might predict sprinting performance, it is necessary to examine which variables are valid enough to be appropriate predictors of soccer-specific sprint performance. However, complex models of adolescent age-specific linear sprinting performance predictors based on either laboratory or field testing are scarce [20,21]. Therefore, the aim of this study was to determine the relationships between linear sprint performance and different variables measured in well-trained young (U19) soccer players.

\section{MATERIAL AND METHODS}

\section{Subjects}

The present study involved young elite male soccer players $(n=18$; age: $17.6 \pm 0.8$ years; body mass: $73.3 \pm$ $5.9 \mathrm{~kg}$; height: $180.8 \pm 4.8 \mathrm{~cm}$ ) playing in the first Czech national U19 soccer league. The study, of a cross-sectional design, was conducted during the pre-season period, which is characterized by a training volume of 6-7 training sessions and 1-2 matches per week. All players abstained from any activities (e.g., highintensity activities) which could disturb the results of the measurement for 48 hours prior to the study. The players were also asked not to use any caffeine beverages (liquid or snack) 4 hours prior to the testing. All participants (or parents or guardians for those under the age of 18) and their coaches received information about the characteristics and purposes of this study, procedures, free participation, the possibility of quitting at any moment, and data confidentiality. The study protocol was approved by the institutional ethics committee, and is consistent with the Declaration of Helsinki. A written informed consent was obtained from all players (or their parents or guardians) before the commencement of the study. Any players with acute medical problems were excluded from the research.

\section{Test procedures}

All measurements were performed on 3 days of a single week (Tuesday, Wednesday, and Friday). On Tuesday, the players performed a set of vertical jumps followed by isokinetic tests of muscular strength of knee flexors and extensors. On Wednesday, the intermittent anaerobic test on a bicycle ergometer $\left(\mathrm{IAnTBE}_{10 \times 6 \mathrm{~s}}\right)$ was conducted, and on Friday, the 20-m linear running sprint test was performed. One week before the test, all athletes were familiarized with the testing procedure except the running sprint test, which was part of the regular training sessions.

\section{Vertical jump performance}

The jump tests were performed on an FP8 force platform (HUR Labs, Tampere, Finland). Before testing, the players completed non-specific warm-up exercises, which included cycling on a stationary bicycle ergometer for $5 \mathrm{~min}$ at $1.5 \mathrm{~W} \cdot \mathrm{kg}^{-1}$, dynamic stretching exercises for $6 \mathrm{~min}$, which targeted the main muscle groups involved during testing, and finally 10 squats with progressive descending. The rest period between the individual trials was $30 \mathrm{~s}$. The following parameters were assessed using the HUR Labs Force Platform Software Suite (HUR Labs, Tampere, Finland): jump height by fly time (cm), maximum power (W), maximum force $(\mathrm{N})$, and maximum force normalized to body weight (relative force [RF], $\mathrm{N} \cdot \mathrm{kg}^{-1}$ ).

Vertical jump performance was measured by means of SqJ and CMJ. During the SqJ, the players squatted down with their hands placed on the hips until the knees were bent at $90^{\circ}$, keeping the trunk in a neutral position, and sustained this position for approximately $1 \mathrm{~s}$. Then they jumped vertically as high as possible. In the CMJ, the players began from the upright position with their hands on the hips, making a downward movement to a $90^{\circ}$ knee angle, and simultaneously performed 2 sets of 5 jumps with a 30 -s rest between the sets. The values of $\mathrm{SqJ}$ and $\mathrm{CMJ}$ with the greatest height $(\mathrm{cm})$ were used for further statistical analyses.

\section{Isokinetic muscular strength of knee flexors and extensors}

Bilateral isokinetic muscular strength of the knee flexors and extensors was measured with the IsoMed 2000 isokinetic dynamometer (D. \& R. Ferstl GmbH, 
Hemau, Germany). The reliability of measurement was confirmed by Dirnberger et al. [22]. The participants were comfortably seated on an adjustable dynamometer chair, with the hip joint at an angle of about $75^{\circ}\left(0^{\circ}=\right.$ full extension $)$. The pelvis and the thigh of the tested leg were fixed by means of straps, and the shoulders were fixed in the ventral-dorsal and cranial-caudal direction by shoulder pads. For further stabilization of the upper body, the players were instructed to hold the handgrips located at the side of the seat during all testing efforts. The axis of rotation of the dynamometer was aligned with the axis of rotation of the knee with the use of the lateral femoral epicondyle as reference. The lever arm of the dynamometer was fixed to the distal part of the shin so that the lower edge of the shin pad was located ca. $2 \mathrm{~cm}$ over the medial apex malleolus. After fixation, a static gravitational correction was applied. The range of motion for testing was set as $10-90^{\circ}$ of knee flexion $\left(0^{\circ}=\right.$ full knee extension). The legs were tested in random order. For the purposes of the results evaluation, the dominant leg was the preferred ball kicking leg.

The testing of the knee extensors and flexors was conducted at an angular velocity of $60^{\circ} \cdot \mathrm{s}^{-1}$ in concentric/concentric and eccentric/eccentric reciprocal actions. The concentric action preceded the eccentric action. Between the tests, there was a rest period of $2 \mathrm{~min}$. Prior to each test, the players performed 2-3 submaximal practice trials as a specific warm-up to become acquainted with the requirements of the test. After the warm-up, the players were instructed to extend or flex the knee with maximum intensity throughout the entire range of motion in each of the subsequent testing repetitions. The testing consisted of a set of 4 reciprocal repetitions. The players were notified by a verbal countdown and were given verbal encouragement and visual online feedback, which was to ensure maximum effort. Both legs were tested according to the same procedure, during which individual settings were automatically activated, rechecked, and adjusted if necessary. The monitored parameters as determined by the IsoMed Analyzer software (D. \& R. Ferstl GmbH, Hemau, Germany) were peak torque $(\mathrm{PT})(\mathrm{N} \cdot \mathrm{m})$, peak work (J), and maximum power (W), also normalized for body weight.

\section{Intermittent anaerobic test on a bicycle ergometer $\left(\operatorname{IAnTBE}_{10 \times 6 s}\right)$}

Intermittent anaerobic performance (repeated sprint ability) was assessed by an all-out intermittent exercise on the Monark 894 Peak Bike bicycle ergometer (Vansbro, Sweden). Prior to the test, a 5-min warm-up was conducted which consisted in pedalling at a moderate frequency on the bicycle ergometer against the resistance of $1.9 \%$ of body weight. Two minutes after the warm-up, the participants executed 2 familiarizations with 6-s cycling sprints of sub-maximum intensity against the resistance of $1.9 \%$ of body weight with 2 -min recovery between the sprints. The test protocol involved 10 6-s cycling sprints against the resistance of $7.5 \%$ of body weight by a mechanical belt brake. The rest interval between 2 consecutive sprints was $30 \mathrm{~s}$. The weight brake resistance dropped down once the pedalling frequency of 120 RPM was achieved. All parts of the testing were performed in the sitting position; the seat height was adjusted for each participant. The standard start position of the pedals was kept with the dominant leg in the upper position. During the test, the participants were encouraged to perform at their maximum. The assessed variables were the relative mechanical peak power $\left(\mathrm{PP}_{\text {IAnTBE10×6s }} ; \mathrm{W} \cdot \mathrm{kg}^{-1}\right)$, measured as the highest mechanical power per 1 revolution across all 6-s bouts of the test, and the highest 6 -s power average in the test as an indicator of maximum anaerobic power $\left(\mathrm{MP}_{6 s} ; \mathrm{W} \cdot \mathrm{kg}^{-1}\right)$.

\section{Linear sprint test}

Before testing, the players underwent a warm-up consisting of a 5-min run at low intensity, followed by 5-min dynamic stretching and 5-min running drills. Afterwards, they performed 1 familiarization with a 20-m sprint. The running sprint test consisted of 2 trials of 20-m maximum sprints on an indoor floorboard surface. The familiarization and testing trials were followed by a 1-min rest interval. The runs were performed from a static biped starting position and the players started from the line placed $50 \mathrm{~cm}$ behind the first photocell. The rest of photocells were placed at $5,10,15$, and $20 \mathrm{~m}$ from the starting line. The sprint trials were recorded by TC Timing System photocells (Brower Timing Systems, Draper, USA). The faster trial was recorded.

\section{Statistical analyses}

The descriptive statistics involved the calculation of the means and standard deviations of the variables. According to the aim of the study, the measures assessed by means of the isokinetic strength test, vertical jump tests, and IAnTBE $_{10 \times 6}$ were the independent variables that were considered possible predictors of performance of a running sprint at 5, 10, 15, and $20 \mathrm{~m}$. To select the independent variables which would best predict the sprint times, the authors carried out mul- 


\section{HUMAN MOVEMENT}

L. Dragula et al., Squat jump as a predictor of sprint in soccer

tiple linear regression (MLR) analysis based on the stepwise forward selection strategy in order to maximize the multiple determination $\left(R^{2}\right)$ for the prediction of sprint performance at each of the 4 distances. The significance of the correlations between the independent and dependent variables was calculated by the Pearson correlation $(\alpha=0.05)$, followed by classification in accordance with Cohen [23]: 0.10 - weak or small association, 0.30 - moderate association, and 0.50 and more - large association. Then, those independent variables that significantly correlated with a given dependent variable were involved in the MLR analysis to reveal regression equations for each of the four dependent variables with the best prediction. The data analyses were performed with the IBM SPSS Statistics programme, version 21.

\section{Ethical approval}

The research related to human use has been complied with all the relevant national regulations, institutional policies and in accordance the tenets of the Helsinki Declaration, and has been approved by the authors' institutional review board or equivalent committee.

\section{RESULTS}

The performance characteristics were summarized and expressed as means and standard deviations (Tables 1 and 2). The correlation analyses showed significant relationships between the times of all sections (distances) of the 20-m sprint and the relative maximum force measured by SqJ, and relative PP measured by IAnTBE $_{10 \times 6 s}$ (Table 3). MP measured by IAnTBE $10 \times 6 \mathrm{~s}$ was associated with the time of the 10-, 15-, and 20-m sprint. The maximum force measured by SqJ was associated with the $20-\mathrm{m}$ sprint, relative PT and relative PP of the dominant limb during eccentric extension measured on the isokinetic dynamometer were associated with the 15-m sprint (Table 3). PT and PP of the non-dominant limb during concentric extension were associated with the 20 -m sprint (Table 3 ).

The multiple regression analyses showed that only the RF measured by SqJ could be used as the 5- and 10-m sprint performance predictor (Table 4), which can explain $18 \%$ of the 5 -m sprint variance and $26 \%$ of the 10-m sprint variance. Furthermore, the MP measured by IAnTBE $10 \times 6 \mathrm{~s}$ with RF measured by $\mathrm{SqJ}$ can be used as the 15- and 20-m sprint performance predictors (Table 4), which can explain $51 \%$ of the $15-\mathrm{m}$ sprint variance and $64 \%$ of the $20-\mathrm{m}$ sprint variance.

Table 1. Soccer players' performance characteristics by linear sprints, countermovement jump, squat jump, and intermittent anaerobic test on a bicycle ergometer (mean $\pm S D$ )

\begin{tabular}{|c|c|c|}
\hline Test & Variable [units] & Performance \\
\hline \multirow{4}{*}{ Linear sprints } & $5 \mathrm{~m}[\mathrm{~s}]$ & $1.10 \pm 0.08$ \\
\hline & $10 \mathrm{~m}[\mathrm{~s}]$ & $1.83 \pm 0.08$ \\
\hline & $15 \mathrm{~m}[\mathrm{~s}]$ & $2.48 \pm 0.09$ \\
\hline & $20 \mathrm{~m}[\mathrm{~s}]$ & $3.09 \pm 0.11$ \\
\hline \multirow{4}{*}{ Countermovement jump } & Jump height by fly time $[\mathrm{cm}]$ & $39.22 \pm 3.40$ \\
\hline & Maximum power [W] & $3996.06 \pm 513.17$ \\
\hline & Maximum force $[\mathrm{N}]$ & $1834.91 \pm 188.59$ \\
\hline & Relative force $\left[\mathrm{N} \cdot \mathrm{kg}^{-1}\right]$ & $24.87 \pm 1.57$ \\
\hline \multirow{4}{*}{ Squat jump } & Jump height by jump time $[\mathrm{cm}]$ & $36.23 \pm 4.75$ \\
\hline & Maximum power [W] & $4133.96 \pm 529.86$ \\
\hline & Maximum force $[\mathrm{N}]$ & $1867.23 \pm 259.15$ \\
\hline & Relative force $\left[\mathrm{N} \cdot \mathrm{kg}^{-1}\right]$ & $25.23 \pm 1.78$ \\
\hline \multirow{2}{*}{ IAnTBE $_{10 \times 6 \mathrm{~s}}$} & Peak power $\left[\mathrm{W} \cdot \mathrm{kg}^{-1}\right]$ & $13.82 \pm 1.02$ \\
\hline & Maximum power $\left[\mathrm{W} \cdot \mathrm{kg}^{-1}\right]$ & $12.03 \pm 0.76$ \\
\hline
\end{tabular}

$\mathrm{IAnTBE}_{10 \times 6 \mathrm{~s}}$ - intermittent anaerobic test on a bicycle ergometer $10 \times 6 \mathrm{~s}$ 
Table 2. Soccer players' isokinetic knee flexion and extension at the velocity of $60^{\circ} \cdot \mathrm{s}^{-1}($ mean $\pm S D)$

\section{Concentric actions}

Knee flexion
Knee extension
Eccentric actions

Knee flexion

DL
NL
Knee extension

DL NL
PT $[N \cdot m]$

$\mathrm{PW}[\mathrm{J}]$

MP $[\mathrm{W}]$

Relative

Relative PW $\left[\mathrm{J} \cdot \mathrm{kg}^{-1}\right]$

Relative MP $\left[\mathrm{W} \cdot \mathrm{kg}^{-1}\right]$

\author{
$147 \pm 20$
}

$147 \pm 20 \quad 144 \pm 20$

NL

$140 \pm 19 \quad 138 \pm 17 \quad 201 \pm 32 \quad 2001 \pm 28 \quad 235 \pm 55$

\begin{tabular}{llll}
$300 \pm 51$ & $174 \pm 28$ & $170 \pm 25$ \\
\hline 5 & $232 \pm 51$ & $168 \pm 29$ & $162 \pm 28$ \\
$317 \pm 54$ & $184 \pm 29$ & $179 \pm 26$
\end{tabular}

$155 \pm 22 \quad 151 \pm 21 \quad 245 \pm 43 \quad 245 \pm 34 \quad 313 \pm 83 \quad 317 \pm 54 \quad 184 \pm 29 \quad 179 \pm 26$

PT - peak torque, PW - peak work, MP - maximum power, DL - dominant lower limb, NL - non-dominant lower limb

Table 3. Correlation matrix between linear sprint and significant laboratory test outcomes

\begin{tabular}{|c|c|c|c|c|c|}
\hline \multirow{2}{*}{ Test } & \multirow{2}{*}{ Outcome [units] } & \multicolumn{4}{|c|}{ Linear sprint time } \\
\hline & & $5 \mathrm{~m}$ & $10 \mathrm{~m}$ & $15 \mathrm{~m}$ & $20 \mathrm{~m}$ \\
\hline \multirow{2}{*}{ Squat jump } & $\mathrm{RMF}\left[\mathrm{N} \cdot \mathrm{kg}^{-1}\right]$ & -0.482 & -0.532 & -0.647 & -0.670 \\
\hline & $\mathrm{MF}[\mathrm{N}]$ & - & - & - & -0.474 \\
\hline \multirow{4}{*}{ Isokinetic strength at $60^{\circ} \cdot \mathrm{s}^{-1}$} & RPT DL EE $\left[\mathrm{N} \cdot \mathrm{m} \cdot \mathrm{kg}^{-1}\right]$ & - & - & -0.431 & - \\
\hline & RPP DL EE $\left[\mathrm{W} \cdot \mathrm{kg}^{-1}\right]$ & - & - & -0.430 & - \\
\hline & PP NL CE [W] & - & - & - & -0.429 \\
\hline & PT NL CE $[\mathrm{N} \cdot \mathrm{m}]$ & - & - & - & -0.428 \\
\hline \multirow{2}{*}{ IAnTBE $_{10 \times 6 s}$} & $\mathrm{PP}\left[\mathrm{W} \cdot \mathrm{kg}^{-1}\right]$ & -0.473 & -0.528 & -0.545 & -0.546 \\
\hline & $\mathrm{MP}_{6 \mathrm{~s}}\left[\mathrm{~W} \cdot \mathrm{kg}^{-1}\right]$ & - & -0.445 & -0.485 & -0.460 \\
\hline
\end{tabular}

The indicated correlations are significant at $p<0.05$.

IAnTBE10 $\times 6$ - intermittent anaerobic test on a bicycle ergometer $10 \times 6 \mathrm{~s}$, RMF - relative maximum force, MF - maximum force, RPT DL EE - relative peak torque of the dominant lower limb during eccentric extension, RPP DL EE - relative peak power of the dominant lower limb during eccentric extension, PP NL CE - peak power of the non-dominant lower limb during concentric extension, PT NL CE - peak torque of the non-dominant lower limb during concentric extension, PP - peak power in the intermittent anaerobic test on a bicycle ergometer, MP6s - maximum 6-s power

Table 4. Multiple regression analysis of sprint time predictors

\begin{tabular}{|c|c|c|c|c|c|c|}
\hline Sprint length & Test & Predictors & $R$ & $R^{2}$ & SEE & $p$ \\
\hline $5 \mathrm{~m}$ & Squat jump & $\mathrm{RF}\left[\mathrm{N} \cdot \mathrm{kg}^{-1}\right]$ & 0.482 & 0.184 & 0.068 & 0.043 \\
\hline $10 \mathrm{~m}$ & Squat jump & $\mathrm{RF}\left[\mathrm{N} \cdot \mathrm{kg}^{-1}\right]$ & 0.549 & 0.258 & 0.064 & 0.018 \\
\hline \multirow{2}{*}{$15 \mathrm{~m}$} & Squat jump & $\mathrm{RF}_{\mathrm{SJ}}\left[\mathrm{N} \cdot \mathrm{kg}^{-1}\right]$ & \multirow{2}{*}{0.647} & \multirow{2}{*}{0.506} & \multirow{2}{*}{0.064} & \multirow{2}{*}{0.040} \\
\hline & IAnTBE $_{10 \times 6 \mathrm{~s}}$ & $\mathrm{PP}\left[\mathrm{W} \cdot \mathrm{kg}^{-1}\right]$ & & & & \\
\hline \multirow{2}{*}{$20 \mathrm{~m}$} & Squat jump & $\mathrm{RF}_{\mathrm{SJ}}\left[\mathrm{N} \cdot \mathrm{kg}^{-1}\right]$ & \multirow{2}{*}{0.769} & \multirow{2}{*}{0.537} & \multirow{2}{*}{0.074} & \multirow{2}{*}{0.038} \\
\hline & IAnTBE $_{10 \times 6 \mathrm{~s}}$ & $\mathrm{PP}\left[\mathrm{W} \cdot \mathrm{kg}^{-1}\right]$ & & & & \\
\hline
\end{tabular}

$\mathrm{IAnTBE}_{10 \times 6 \mathrm{~s}}$ - intermittent anaerobic test on a bicycle ergometer $10 \times 6 \mathrm{~s}$, RF - relative force, $\mathrm{RF}_{\mathrm{SJ}}$ - relative force in the squat jump, $\mathrm{PP}$ - peak power in the intermittent anaerobic test on a bicycle ergometer 


\section{DISCUSSION}

The main finding of this investigation is that the multiple regression analysis showed $\mathrm{RF}$ in $\mathrm{SqJ}$ as the strongest predictor of sprint performance over $5 \mathrm{~m}$ and $10 \mathrm{~m}$. Together, $\mathrm{RF}$ in $\mathrm{SqJ}$ and relative PP in $\mathrm{IAnTBE}_{10 \times 6 \mathrm{~s}}$ seem to be good predictors of sprint over $15 \mathrm{~m}$ and $20 \mathrm{~m}$. This result is in agreement with West et al. [24], who found out that strength relative to the participant's body weight rather than absolute force output contributed to models of linear sprint times and predicted short 10-m linear sprint performance. The finding is also in accordance with a previous regression model [25], in which sprint performance variance was explained by relative maximum strength along with power values and rate of force development in loaded SqJ. The relationship between SqJ, PP in IAnTBE $\mathrm{I}_{10 \times 6 \mathrm{~s}}$, and short sprint performance might be explained by their similarities in the knee extension action to provide propulsion for movement acceleration.

The results of the present study did not confirm that sprint performance could be predicted by jump height $[8,10]$, but rather by SqJ RF. The authors believe that the results are caused by the different orientation of the reactive force vector, as the RF production seems to be similar during both measured movements, but the force vector is oriented vertically during SqJ and inclined in the horizontal plane during sprint. Furthermore, the correlation analyses showed that the relationship between $\mathrm{SqJ} R F$ and linear sprint increased along with the sprint distance of 5-20 m (Table 3). The finding that sprint time over a longer distance has a stronger relationship to the amount of force during $\mathrm{SqJ}$ compared with a short distance corresponds with the findings of a previous study [26], where CMJ was proved an appropriate test when predicting 30-100-m sprint performance.

The authors of the present paper also found a stronger relationship between PP per 1 revolution $\left(\mathrm{W} \cdot \mathrm{kg}^{-1}\right)$ in $\mathrm{IAnTBE}_{10 \times 6 \mathrm{~s}}$ and sprint performance over 5, 10, 15, and $20 \mathrm{~m} . \mathrm{MP}_{6 \mathrm{~s}}\left(\mathrm{~W} \cdot \mathrm{kg}^{-1}\right)$ in this test significantly correlated with sprint performance over 10,15 , and $20 \mathrm{~m}$. The results of this study indicated that $\mathrm{IAnTBE}_{10 \times 6 \mathrm{~s}}$ was only a moderate predictor of run times, but a stronger predictor when the measured values were adjusted for body weight. The similar relationship between sprint performance and anaerobic power was previously tested by Tharp et al. [27], who found a moderate correlation between power output (5 s) in the Wingate anaerobic test (WAnT) and 50-yard run $(45.72 \mathrm{~m})(r=$ -0.53 ) performed by volunteers aged $10-15$ years, and observed that values adjusted for body weight were stronger predictors of sprint performance than max- imal PP values. On the other hand, Alemdaroğlu [28] reported a significant relationship between absolute peak power output in WAnT and a 10-m sprint $(r=$ -0.52 ) and an insignificant relationship between mean power in absolute values and a 10-m sprint. Another study [29] demonstrated significant correlations between relative PP in WAnT and a sprint over $50 \mathrm{~m}(r=$ $-0.70)$ and $200 \mathrm{~m}(r=-0.54)$. The study suggested that $\mathrm{PP}$ in relative values and in WAnT could be used as a predictor of sprint performance, the strength of WAnT predictability depending, however, on sprint length. The findings of the present study suggest that power outputs in relative values are good predictors of sprint performance at short distances.

The results of this study show an insignificant relationship between single-joint isokinetic muscle strength at a low velocity $\left(60^{\circ} \cdot \mathrm{s}^{-1}\right)$ and speed performance at 5,10 , and $20 \mathrm{~m}$ in relative values. On the other hand, the authors found a moderate correlation between relative PT and relative $\mathrm{PP}$ of the dominant leg during eccentric action and 15-m sprint performance. Absolute values of PT during concentric action and $\mathrm{PP}$ of the non-dominant leg were associated only with the 20 -m sprint performance. The findings only partly coincide with the results of a study by Lehance et al. [30], who observed an association between a $10-\mathrm{m}$ sprint and knee flexor $(r=-0.48)$ and extensor strength $(r=-0.51)$ in concentric action at the angular velocity of $60^{\circ} \cdot \mathrm{s}^{-1}$, and suggested that isokinetic muscle strength at the velocity of $60^{\circ} \cdot \mathrm{s}^{-1}$, which is generally considered an indicator of maximum strength, was a weak predictor of linear sprint performance. Thus, for the purpose of linear sprint performance prediction, it seems more efficient to use isokinetic testing at higher angular velocities [15, 16, 31], which better corresponds with the higher speed demands on muscle action in linear sprinting in soccer.

A limitation of the present study is the range of the measured isokinetic force speed, as higher angular velocities of $180^{\circ} \cdot \mathrm{s}^{-1}$ and $240^{\circ} \cdot \mathrm{s}^{-1}$ were not used. The reason for the measurement using the angular velocity of $60^{\circ} \cdot \mathrm{s}^{-1}$ was to apply the most commonly measured velocity, which allows the measurement of maximum muscle strength [32]. Another limitation is the absence of a soccer player agility test, which is more useful for soccer performance [33] and includes changes in directional speed along with short sprints.

\section{CONCLUSIONS}

When presenting the results of laboratory tests to coaches or strength and fitness practitioners, RF in SqJ could be used as a parameter relevant to linear 5-, 
10-, 15-, and 20-m sprint. Together with RF in SqJ, PP in $\mathrm{IAnTBE}_{10 \times 6 \mathrm{~s}}$ can be used as a parameter relevant to linear 15- and 20-m sprint. Laboratory examiners should not associate single joint isokinetic force, jump height, jump power, or anaerobic capacity with linear sprint performance in U19 elite soccer players.

\section{Acknowledgements}

The authors would like to thank the soccer club SK Sigma Olomouc for their participation in the research, especially the coaches and players of the U19 category in the 2016/2017 season. The study was supported by a research grant IGA_FTK_2017_010 of Palacky University, Olomouc, Czech Republic and by grant UNCE032 at Charles University, Prague, Czech Republic.

\section{Disclosure statement}

No author has any financial interest or received any financial benefit from this research.

\section{Conflict of interest}

Authors state no conflict of interest.

\section{References}

1. Lipińska P, Szwarc A. Laboratory tests and game performance of young soccer players. TRENDS Sport Sci. 2016;23(1):33-39.

2. Dellal A, Chamari K, Wong DP, Ahmaidi S, Keller D, Barros R, et al. Comparison of physical and technical performance in European soccer match-play: FA Premier League and La Liga. Eur J Sport Sci. 2011;11(1):5159; doi: 10.1080/17461391.2010.481334.

3. Bangsbo J. The physiology of soccer - with special reference to intense intermittent exercise. Acta Physiol Scand Suppl. 1994;619:1-155.

4. Mohr M, Krustrup P, Bangsbo J. Match performance of high-standard soccer players with special reference to development offatigue. J Sports Sci. 2003;21(7):519_ 528; doi: 10.1080/0264041031000071182.

5. Reilly T, Williams AM, Nevill A, Franks A. A multidisciplinary approach to talent identification in soccer. J Sports Sci.2000;18(9):695-702; doi:10.1080/0264041 0050120078.

6. Comfort P, Bullock N, Pearson SJ. A comparison of maximal squat strength and 5-, 10-, and 20-meter sprint times, in athletes and recreationally trained men. J Strength Cond Res. 2012;26(4):937-940; doi: 10.1519/ JSC.0b013e31822e5889.

7. McBride JM, Blow D, Kirby TJ, Haines TL, Dayne AM, Triplett NT. Relationship between maximal squat strength and five, ten, and forty yard sprint times. J Strength Cond Res. 2009;23(6):1633-1636; doi: 10.1519/JSC.0b013e 3181b2b8aa.
8. Wisløff U, Castagna C, Helgerud J, Jones R, Hoff J. Strong correlation of maximal squat strength with sprint performance and vertical jump height in elite soccer players. Br J Sports Med. 2004;38(3):285-288; doi: 10.1136/bjsm.2002.002071.

9. Jonathan N, Russell M, Shearer D, Cook C, Kilduff L. Predictors of linear and multidirectional acceleration in elite soccer players. J Strength Cond Res. 2017; doi: 10.1519/JSC.0000000000001897.

10. Comfort P, Stewart A, Bloom L, Clarkson B. Relationships between strength, sprint, and jump performance in well-trained youth soccer players. J Strength Cond Res. 2014;28(1):173-177; doi: 10.1519/JSC.0b013e318291b8c7.

11. Yanci J, Los Arcos A, Mendiguchia J, Brughelli M. Relationships between sprinting, agility, one- and twoleg vertical and horizontal jump in soccer players. Kinesiology. 2014;46(2):194-201.

12. Loturco I, Pereira LA, Kobal R, Maldonado T, Piazzi AF, Bottino A, et al. Improving sprint performance in soccer: effectiveness of jump squat and Olympic push press exercises. PLoS One. 2016;11(4); doi: 10.1371/journal.pone.0153958.

13. López-Segovia M, Marques MC, Tillaar van den R, González-Badillo JJ. Relationships between vertical jump and full squat power outputs with sprint times in U21 soccer players. J Hum Kinet. 2011;30:135-144; doi: 10.2478/v10078-011-0081-2.

14. Bračič M, Hadžič V, Čoh M, Derviševič E. Relationship between time to peak torque of hamstrings and sprint running performance. Isokinet Exerc Sci. 2011;19(4): 281-286; doi: 10.3233/IES-2011-0426.

15. Cotte T, Chatard J-C. Isokinetic strength and sprint times in English Premier League football players. Biol Sport. 2011;28(2):89-94; doi: 10.5604/942736.

16. Newman MA, Tarpenning KM, Marino FE. Relationships between isokinetic knee strength, single-sprint performance, and repeated-sprint ability in football players. J Strength Cond Res. 2004;18(4):867-872; doi: 10.1519/13843.1.

17. Jönhagen S, Ericson MO, Németh G, Eriksson E. Amplitude and timing of electromyographic activity during sprinting. Scand J Med Sci Sports. 1996;6(1):15-21; doi: 10.1111/j.1600-0838.1996.tb00064.x.

18. Wiemann K, Tidow G. Relative activity of hip and knee extensors in sprinting - implications for training. New Stud Athl. 1995;10(1):29-49.

19. Mero A, Komi PV. Electromyographic activity in sprinting at speeds ranging from sub-maximal to supra-maximal. Med Sci Sports Exerc. 1987;19(3):266-274; doi: 10.1249/00005768-198706000-00014.

20. Malý T, Zahálka F, Malá L, Teplan J. Profile, correlation and structure of speed in youth elite soccer players. J Hum Kinet. 2014;40(1):149-159; doi: 10.2478/ hukin-2014-0017.

21. Malý T, Zahálka F, Hráský P, Malá L, Ižovská J, Bujnovský D, et al. Age-related differences in linear sprint 


\section{HUMAN MOVEMENT}

L. Dragula et al., Squat jump as a predictor of sprint in soccer

and power characteristics in youth elite soccer players. J Phys Educ Sport. 2015;15(4):857-863; doi: 10.7752/ jpes.2015.04132.

22. Dirnberger J, Wiesinger H-P, Kösters A, Müller E. Reproducibility for isometric and isokinetic maximum knee flexion and extension measurements using the IsoMed 2000-dynamometer. Isokinet Exerc Sci. 2012; 20(3):149-153; doi: 10.3233/IES-2012-0451.

23. Cohen J. Statistical power analysis for the behavioral sciences, $2^{\text {nd }}$ ed. Hillsdale: Lawrence Erlbaum Associates; 1988.

24. West DJ, Owen NJ, Jones MR, Bracken RM, Cook CJ, Cunningham DJ, et al. Relationships between forcetime characteristics of the isometric midthigh pull and dynamic performance in professional rugby league players. J Strength Cond Res. 2011;25(11):3070-3075; doi: 10.1519/JSC.0b013e318212dcd5.

25. Switon PA, Lloyd R, Keogh JWL, Agouris I, Stewart AD. Regression models of sprint, vertical jump, and change of direction performance. J Strength Cond Res. 2014;28(7):1839-1848; doi: 10.1519/JSC.000000000 0000348 .

26. Bret C, Rahmani A, Dufour A-B, Messonnier L, Lacour J-R. Leg strength and stiffness as ability factors in 100-m sprint running. J Sports Med Phys Fitness. 2002;42(3):274-281.

27. Tharp GD, Newhouse RK, Uffelman L, Thorland WG, Johnson GO. Comparison of sprint and run times with performance on the Wingate anaerobic test. Res Q Exerc Sport.1985;56(1):73-76; doi: 10.1080/02701367.1985. 10608434.

28. Alemdaroğlu U. The relationship between muscle strength, anaerobic performance, agility, sprint ability and vertical jump performance in professional basketball players. J Hum Kinet. 2012;31(1):149-158; doi: 10.2478/v10078-012-0016-6.

29. Patton JF, Duggan A. An evaluation of tests of anaerobic power. Aviat Space Environ Med. 1987;58(3):237-242.

30. Lehance C, Binet J, Bury T, Croisier JL. Muscular strength, functional performances and injury risk in professional and junior elite soccer players. Scand J Med Sci Sports. 2009;19(2):243-251; doi: 10.1111/j.1600-0838.2008. 00780.x.

31. Dowson MN, Nevill ME, Lakomy HKA, Nevill AM, Hazeldine RJ. Modelling the relationship between isokinetic muscle strength and sprint running performance. J Sports Sci. 1998;16(3):257-265; doi: 10.1080/0264 04198366786.

32. Tourny-Chollet C, Leroy D, Léger H, Beuret-Blanquart F. Isokinetic knee muscle strength of soccer players according to their position. Isokinet Exerc Sci. 2000;8(4):187193.

33. Hojka V, Stastny P, Rehak T, Gołas A, Mostowik A, Zawart $\mathrm{M}$, et al. A systematic review of the main factors that determine agility in sport using structural equation modeling. J Hum Kinet. 2016;52(1):115-123; doi: 10.1515/hukin-2015-0199. 